\title{
Potencial bioativo dos análogos sintéticos das riparinas: uma revisão sistemática
}

\author{
Bioactive potential of riparina synthetic analogs: a systematic review \\ Potencial bioactivo de los análogos sintéticos de riparina: una revisión sistemática
}

Recebido: 18/11/2021 | Revisado: 26/11/2021 | Aceito: 03/12/2021 | Publicado: 07/12/2021

\author{
Duanne Mendes Gomes \\ ORCID: https://orcid.org/0000-0003-1506-2055 \\ Universidade Federal do Piauí, Brasil \\ E-mail: duannemendesg@gmail.com \\ Paulo Monteiro Araujo \\ ORCID: https://orcid.org/0000-0002-5829-6268 \\ Universidade Federal do Piauí, Brasil \\ E-mail: paulomonteirothe@gmail.com \\ Sávio Freire da Silva \\ ORCID: https://orcid.org/0000-0002-6221-6910 \\ Universidade Federal do Piauí, Brasil \\ E-mail: saviofreire.silva@gmail.com \\ Veridiana Rebelo dos Santos \\ ORCID: https://orcid.org/0000-0002-1118-5392 \\ Universidade Federal do Piauí, Brasil \\ E-mail: veridianarebelo@gmail.com \\ Stanley Juan Chavez Gutierrez \\ ORCID: https://orcid.org/0000-0002-9353-1307 \\ Universidade Federal do Piauí, Brasil \\ E-mail: stanleychavez@ufpi.edu.br \\ Lyghia Maria Araújo Meirelles \\ ORCID: https://orcid.org/0000-0002-0174-4955 \\ Centro Universitário Santo Agostinho, Brasil \\ E-mail: lyghiamaria@unifsa.com.br \\ Maria das Graças Freire de Medeiros Carvalho \\ ORCID: https://orcid.org/0000-0002-6161-7085 \\ Universidade Federal do Piauí, Brasil \\ E-mail:mgfmedeiros@ hotmail.com
}

\begin{abstract}
Resumo
Objetivo: Realizar uma revisão sistemática dos estudos com os análogos sintéticos da Aniba riparia, as riparinas A, B, C, D, E e F, com o intuito de evidenciar os potenciais farmacológicos já elucidados. Metodologia: Utilizou-se três bases de dados para seleção dos artigos a serem incluídos na revisão Embase, Pubmed e Web of Science, com a seguinte estratégia de busca simplificada (Riparin or Aniba riparia), ao final da seleção, resultou em 10 artigos para a elaboração deste trabalho. Resultados: As publicações dos estudos com derivados sintéticos foram identificadas entre os anos de 2014 a 2021. Sendo que a maioria das pesquisas utilizavam a riparina A, seguida pela B, e todos tratavam-se de estudos pré-clínicos. Foram relatados importantes efeitos farmacológicos, como as atividades antibacteriana, antifúngica, antiinflamatória, antinociceptiva, antitumoral, imunomoduladora, antiprotozoário, antiparasitária, ansiolítica e antioxidante. Discussão: Os estudos realizados com os análogos sintéticos têm colocado as mesmas como possíveis ferramentas terapêuticas, considerando as importantes atividades farmacológicas já relatadas. Conclusão: Contudo, considerando que a literatura já relata seis riparinas sintéticas, o número de artigos publicados voltados para elucidação de possíveis atividades farmacológicas parece reduzido, mesmo sendo promissoras candidatas para o desenvolvimento de pesquisas direcionadas para descobertas de novos fármacos.
\end{abstract}

Palavras-chave: Aniba riparia; Bioprospecção; Ações farmacológicas.

\begin{abstract}
Objective: To carry out a systematic review of studies with synthetic analogues of Aniba riparia, riparins A, B, C, D, E and $\mathrm{F}$, in order to highlight the pharmacological potentials already elucidated. Methodology: Three databases were used to select the articles to be included in the Embase, Pubmed and Web of Science review, with the following simplified search strategy (Riparin or Aniba riparia), at the end of the selection, resulting in 10 articles for the elaboration of this work. Results: Publications of studies with synthetic derivatives were identified between the years 2014 to 2021 . Most of the researches used riparin A, followed by B, and all were pre-clinical studies. Important pharmacological effects have been reported, such as antibacterial, antifungal, anti-inflammatory, antinociceptive, antitumor, immunomodulatory, antiprotozoal, antiparasitic, anxiolytic and antioxidant activities. Discussion: Studies carried out with synthetic analogues have placed them as possible therapeutic tools, considering the important pharmacological activities already reported. Conclusion: However, considering that the literature already reports six synthetic riparins,
\end{abstract}


the number of articles published aimed at elucidating possible pharmacological activities seems small, even though they are promising candidates for the development of research aimed at discovering new drugs.

Keywords: Aniba riparia; Bioprospecting; Pharmacologic actions.

\section{Resumen}

Objetivo: Realizar una revisión sistemática de estudios con análogos sintéticos de Aniba riparia, riparinas A, B, C, D, E y F, con el fin de resaltar los potenciales farmacológicos ya dilucidados. Metodología: Se utilizaron tres bases de datos para seleccionar los artículos a incluir en la revisión Embase, Pubmed y Web of Science, con la siguiente estrategia de búsqueda simplificada (Riparin o Aniba riparia), al final de la selección, resultando en 10 artículos para la elaboración de este trabajo. Resultados: Se identificaron publicaciones de estudios con derivados sintéticos entre los años 2014 a 2021. La mayoría de las investigaciones utilizaron riparina A, seguida de B, y todas fueron estudios preclínicos. Se han reportado efectos farmacológicos importantes, tales como actividades antibacterianas, antifúngicas, antiinflamatorias, antinociceptivas, antitumorales, inmunomoduladoras, antiprotozoarias, antiparasitarias, ansiolíticas y antioxidantes. Discusión: Los estudios realizados con análogos sintéticos los han colocado como posibles herramientas terapéuticas, considerando las importantes actividades farmacológicas ya reportadas. Conclusión: Sin embargo, considerando que la literatura ya reporta seis riparinas sintéticas, el número de artículos publicados para dilucidar posibles actividades farmacológicas es pequeño, aunque son candidatos prometedores para el desarrollo de investigaciones dirigidas al descubrimiento de nuevos fármacos.

Palabras clave: Aniba riparia; Bioprospección; Acciones farmacológicas.

\section{Introdução}

Os estudos com produtos naturais e seus análogos estruturais têm mostrado ao longo dos anos, uma grande contribuição para a farmacoterapia, especialmente para o câncer e doenças infecciosas, impulsionando assim a exploração dos recursos naturais, a fim de descobrir novas entidades químicas com aplicabilidades. Estes estudos têm demonstrados resultados promissores no desenvolvimento de novos fármacos, resultando em um número significativo de medicamentos derivados de produtos naturais, possibilitando assim a inovação e viabilização de tratamentos disponíveis na clínica (Atanasov et al., 2021; Newman \& Cragg, 2020).

Dentro desse contexto de utilização de recursos naturais para descoberta de novos medicamentos, destaca-se o uso da espécie Aniba riparia (Nees) Mez, que pertence à família Lauraceae, popularmente conhecido no Brasil como "louro", onde os primeiros registros de utilização desta família datam de 2.800 anos a.C (Marques, 2001). Sendo primordialmente encontrado na Amazônia Central e Guiana (Barbosa Filho et al., 1990; Castelo-Branco et al., 2000). Em pesquisas iniciais, com o fruto verde da planta, foram encontradas uma variedade de substâncias, com destaque para as alcamidas, esta constitui uma importante classe de alcaloides, contendo uma função amida restritas a poucos produtos naturais (Barbosa Filho et al., 1990; Catão et al., 2005).

As alcamidas são um grupo diferenciado de produtos naturais que são formadas pela conjugação de diferentes rotas metabólicas, consideradas metabólitos secundários, cuja estrutura geral é originada da condensação de um ácido graxo insaturado e uma amina, resultando em uma amida (alcamida). São consideradas bioativas, ou seja, uma pequena quantidade apresenta uma resposta notável nas células receptoras (Hofer et al., 1986; Torres \& Chavez, 2001).

As riparinas (Rips), alcaloides naturais do grupo alcamida, podem também ser sintetizadas por métodos simples, potencializando a sua aplicação no âmbito farmacêutico. Desde que a rota sintética se tornou a principal forma de obter moléculas deste grupo de compostos, vários derivados sintéticos foram sintetizados denominados riparinas A e B, e a partir da condensação de ésteres metílicos com as feniletilaminas substituídas, sintetizou as riparinas C, D, E e F. Esses análogos estruturais podem ser preparados utilizando metodologia fundamentada na reação Schotten-Baumann (Barbosa Filho et al., 1990; Catão et al., 2005; Gutierrez, 2006; Gutierrez et al., 2005; Nascimento et al., 2016).

Diversos efeitos biológicos já foram elucidados utilizando tanto extratos de frutos e cálices, como dos constituintes isolados e análogos sintéticos. Ação antitumoral e leishmanicida (Araújo et al., 2016), em outro estudo evidenciou-se propriedades antitumorais apresentando taxas de inibição do crescimento celular superiores a 70\% (Nunes et al., 2014), efeitos antinociceptivos (Nascimento et al., 2016), potencialidades do uso das riparina no tratamento de doenças neurodegerativas e 
inflamatórias. Pois, demonstrou ação neuroprotetora (Nunes et al., 2015), efeito antioxidante, diminuindo a produção de citocinas pró-inflamatórias e do estresse oxidativo (Silva et al., 2015), efeito bactericida para algumas bactérias Gram-positivas e Gramnegativas, fungicida contra Candida spp. (Costa et al., 2021b).

Com isso, o objetivo desta pesquisa foi realizar uma compilação dos estudos relacionados às riparinas A, B, C, D, E e F (Rips A-F) e apresentar uma visão geral do estado atual de desenvolvimento das pesquisas relacionadas a elas.

\section{Metodologia}

Trata-se uma revisão sistemática que buscou artigos nos bancos de dados: PubMed $^{\circledR}$, Embase $^{\circledR}$ e Web of Science ${ }^{\mathrm{TM}}$, visando primordialmente responder a seguinte pergunta: Quais os potenciais efeitos farmacológicos já elucidados para os análogos sintéticos derivados da Aniba riparia?

Realizou-se a pesquisa nas bases em 18 de agosto de 2021, às 12:00 h, utilizando a seguinte estratégia de busca: PubMed - "riparin" OR "aniba riparia", Embase - 'riparin' OR 'aniba riparia' e Web of Science - "riparin" (All Fields) or "aniba riparia" (All Fields). Sendo selecionados para a próxima etapa, todos os artigos encontrados por essa estratégia de busca, atemporal.

\subsection{Filtragem dos artigos}

$\mathrm{Na}$ filtragem inicial, avaliou-se o título e o resumo dos artigos, a fim de determinar se eles utilizavam de alguma forma os análogos sintéticos da Aniba riparia.

Posteriormente, foram incluídos os artigos que avaliavam uma possível aplicação farmacológica das riparinas A-F e que estavam disponíveis em sua forma completa.

\subsection{Extração e síntese dos dados}

Os investigadores extraíram dos artigos os seguintes dados: autor, ano de publicação, riparina estudada, escala do estudo, objetivo da pesquisa e atividade farmacológica.

\section{Resultados}

Inicialmente na pesquisa, foram encontrados 160 artigos $\left(43\right.$ Pubmed $^{\circledR}$ e 54 Embase $^{\circledR}$ e 63 Web of Science ${ }^{\mathrm{TM}}$ ), dos quais, 85 estavam repetidos nas bases. Em seguida foram excluídos 64 por não tratarem dos derivados sintéticos, as riparinas A, B, C, D, E e F, ficando apenas 11 artigos para a leitura na íntegra. Na fase de leitura integral do texto, 1 (um) artigo foi excluído, pois não avaliava a atividade farmacológica das riparinas A-F (Araújo et al., 2018). Ao final da aplicação da estratégia, 10 artigos foram incluídos nessa síntese (Figura 1). 
Research, Society and Development, v. 10, n. 16, e328101623510, 2021

(CC BY 4.0) | ISSN 2525-3409 | DOI: http://dx.doi.org/10.33448/rsd-v10i16.23510

Figura 1 - Fluxograma de seleção dos artigos recuperados pelas estratégias de busca.

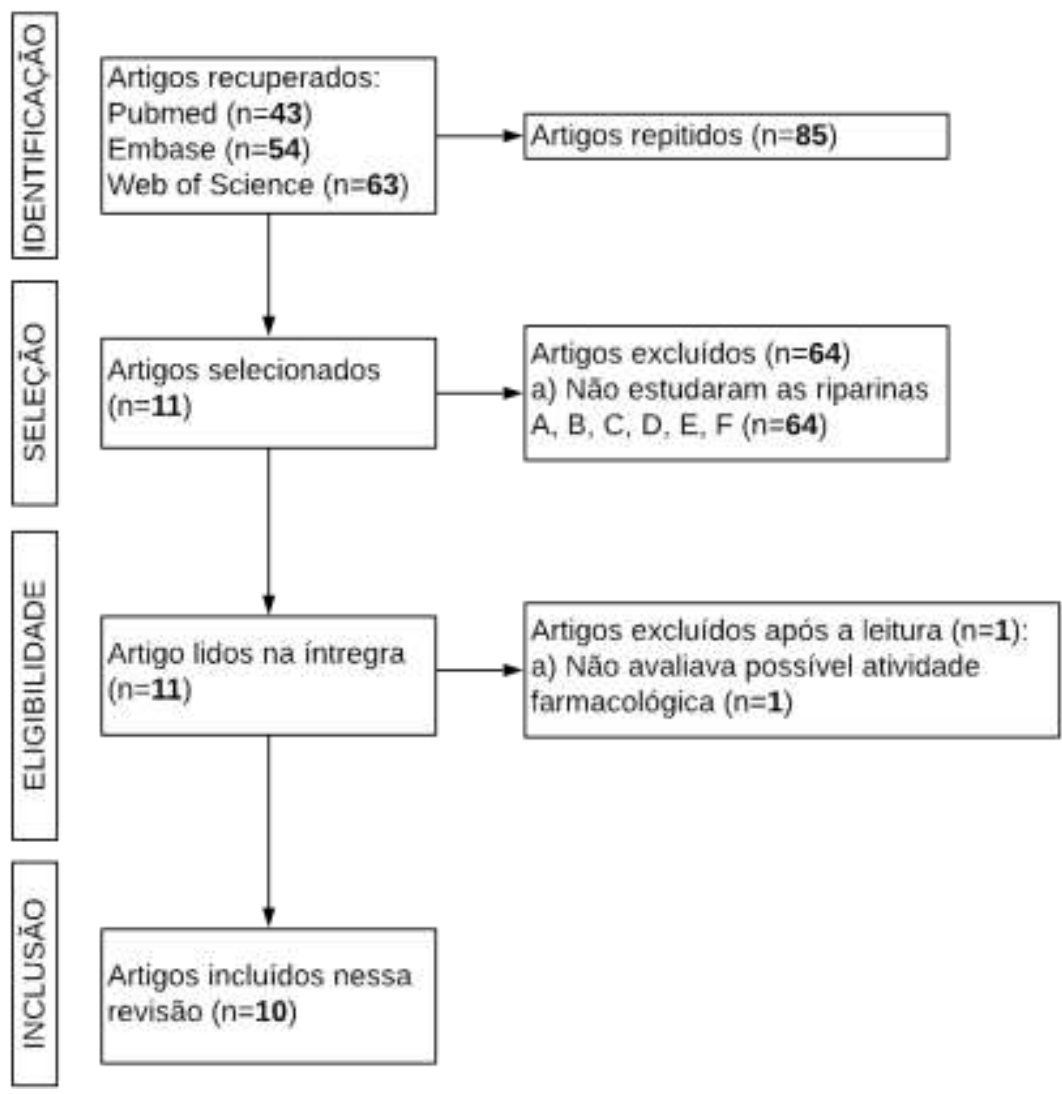

Fonte: Autores.

O período de tempo das publicações está apresentado no Gráfico 1, abrangeu estudos de 2014 a agosto de 2021, o volume maior foi no ano de 2015, seguidos de 2016, 2018, 2021 e 2014.

Gráfico 1 - Distribuição das publicações referentes a análogos sintéticos da A. riparia, publicados até 17 de agosto de 2021, nas bases PubMed, Embase e Web of Science.

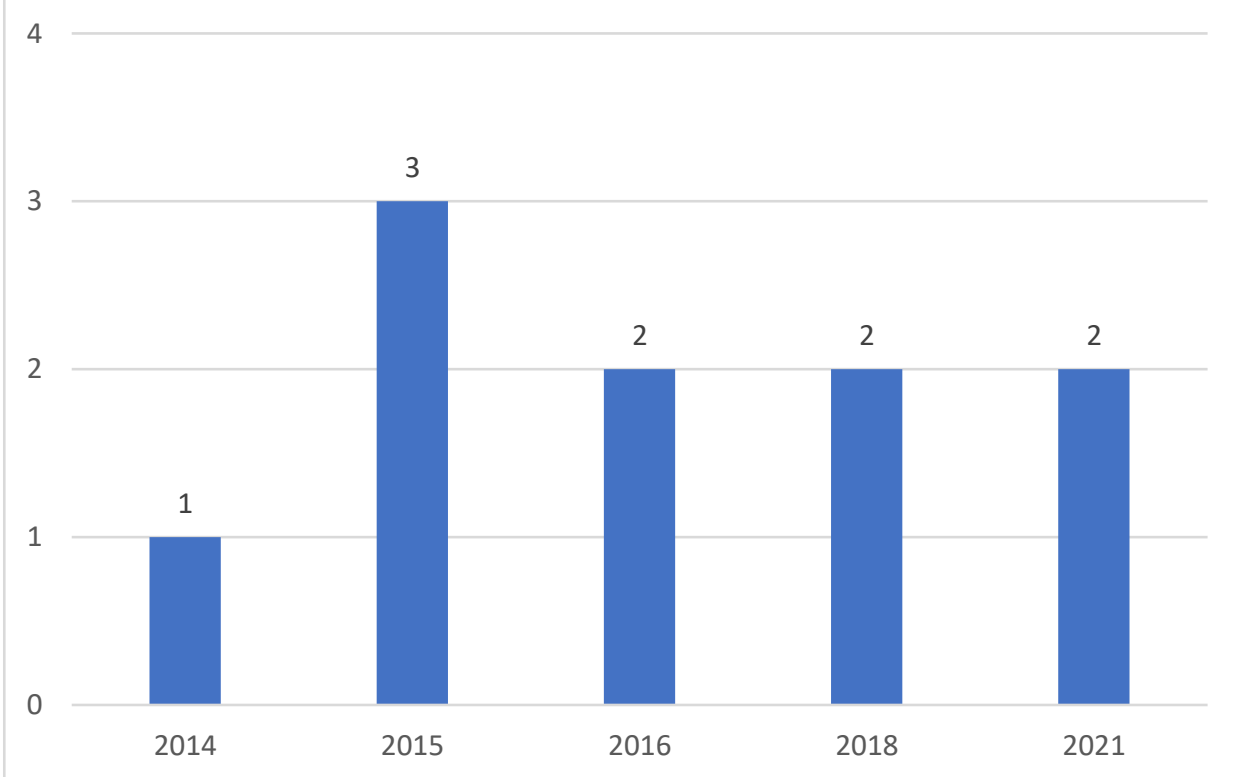

Fonte: Autores. 
Com a análise dos estudos selecionados foi possível ter uma visão sobre as pesquisadas realizadas com os análogos sintéticos como também os tipos de experimentos envolvidos para avaliação das atividades bioativas, a seguir apresentadas na Tabela 1.

Tabela 1 - Síntese dos artigos selecionados quanto ao tipo de riparina sintética estuda, escala do estudo, principais objetivos propostos e os autores.

\begin{tabular}{|c|c|c|c|}
\hline $\begin{array}{l}\text { (AUTOR, } \\
\text { ano) }\end{array}$ & $\begin{array}{l}\text { Riparinas } \\
\text { estudadas }\end{array}$ & $\begin{array}{l}\text { Escala do } \\
\text { estudo }\end{array}$ & Objetivo de estudo \\
\hline $\begin{array}{l}\text { (Nunes et al., } \\
2014)\end{array}$ & $\begin{array}{l}\mathrm{A}, \mathrm{B}, \mathrm{C}, \mathrm{D}, \mathrm{E} \mathrm{e} \\
\mathrm{F}\end{array}$ & In vitro & Avaliar atividade antioxidante e a toxicidade sobre linhagem de células tumorais. \\
\hline $\begin{array}{l}\text { (Nunes et al., } \\
\text { 2015) }\end{array}$ & A & $\begin{array}{l}\text { In vivo e in } \\
\text { vitro }\end{array}$ & $\begin{array}{l}\text { Avaliou o estresse oxidativo em mitocôndrias e a atividade locomotora e } \\
\text { miorrelaxante. }\end{array}$ \\
\hline $\begin{array}{l}\text { (Santiago et } \\
\text { al., 2015) }\end{array}$ & B & In vivo & Avaliou o efeito em modelo de inflamação e dor e a atividade antioxidante. \\
\hline $\begin{array}{l}\text { (Silva et al., } \\
\text { 2015) }\end{array}$ & A & In vivo & Avaliou a atividade anti-inflamatória. \\
\hline $\begin{array}{l}\text { (Araújo et al., } \\
\text { 2016) }\end{array}$ & A & In vitro & Avaliou a atividade antioxidante, antitumoral e leishmanicida. \\
\hline $\begin{array}{l}\text { (Costa et al., } \\
\text { 2016) }\end{array}$ & $\mathrm{A}, \mathrm{B}, \mathrm{C}, \mathrm{D}$ e $\mathrm{E}$ & In vitro & $\begin{array}{l}\text { Avaliou a atividade antimicrobiana dos derivados sintéticos sozinhos e em combinação } \\
\text { com antimicrobianos (Fluoroquinolonas) contra cepas se } S \text {. aures que superexpressam } \\
\text { a NorA. }\end{array}$ \\
\hline $\begin{array}{l}\text { (Araújo et al., } \\
\text { 2018) }\end{array}$ & A & $\begin{array}{l}\text { In sílico e } \\
\text { in vivo }\end{array}$ & $\begin{array}{l}\text { Avaliou a toxidade oral aguda em camundongos e o efeito ansiolítico por modelos } \\
\text { experimentais e computacionais. }\end{array}$ \\
\hline $\begin{array}{l}\text { (Mafud et al., } \\
\text { 2018) }\end{array}$ & $\begin{array}{l}\mathrm{A}, \mathrm{B}, \mathrm{C}, \mathrm{D}, \mathrm{E} \text { e } \\
\mathrm{F}\end{array}$ & $\begin{array}{l}\text { In sílico e } \\
\text { ex vivo }\end{array}$ & Avaliou o efeito esquistossomicida e o perfil farmacêutico, estrutural e toxicológico. \\
\hline $\begin{array}{l}\text { (Costa et al., } \\
\text { 2021a) }\end{array}$ & $\mathrm{E}$ & In vitro & Avaliou o efeito antileishmania e a atividade imunomoduladora. \\
\hline $\begin{array}{l}\text { (Costa et al., } \\
\text { 2021b) }\end{array}$ & A e E & In vitro & Avaliou o efeito antimicrobiano. \\
\hline
\end{tabular}

Fonte: Elaborado pelos autores baseada nos artigos citados.

Entre as riparinas investigadas, observou-se um maior volume de artigos com a riparina A (Rip-A), seguido pela riparina B (Rip-B). Esses estudos objetivaram pela busca dos possíveis efeitos farmacológicos e toxicológicos, através de modelos "in sílico", "in vitro", "ex vivo" e "in vivo" utilizando modelos animais, especialmente envolvendo roedores, com uma diversidade de metodologias aplicadas conforme os objetivos pretendidos.

Importantes atividades e efeitos farmacológicos foram pesquisados, tendo resultados promissores elucidados, conforme a Tabela 2. A Rip-A mostrou um potencial antibacteriano modulador, propriedades antiinflamatórias, antinociceptivo, ansiolítica, antioxidante, antitumoral e leishmanicida (Araújo et al., 2016, 2018; Costa et al., 2016; Nunes et al., 2014, 2015; Silva et al., 2015).

A Rip-B, apresentou propriedades antibacterianas por efeito modulador, antiinflamatórias, antinociceptivo, antioxidante e antiparasitária (Costa et al., 2016; Mafud et al., 2018; Nunes et al., 2014; Santiago et al., 2015). As riparinas C e D mostraram atividade antibacteriana intrínseca e moduladora, antioxidante e antitumoral (Costa et al., 2016; Nunes et al., 2014). A riparina E (Rip-E) atividade antibacteriana intrínseca e moduladora, antifungica, antioxidante, antiparasitária, antitumoral e imunomoduladora (Costa et al., 2016; Costa et al., 2021a; Costa et al., 2021b; Mafud et al., 2018; Nunes et al., 2014) e a riparina F (Rip-F) demonstrou possuir efeito antioxidante, antiparasitário e antitumoral (Mafud et al., 2018; Nunes et al., 2014). 
Tabela 2 - Principais efeitos e atividades elucidados dos derivados análogos sintéticos das riparinas.

\begin{tabular}{|c|c|c|c|c|c|c|}
\hline \multirow[t]{2}{*}{ Atividade } & \multicolumn{6}{|c|}{ Riparinas } \\
\hline & A & B & C & D & $\mathbf{E}$ & F \\
\hline Antibacteriana (Intrínseco) & & & $\begin{array}{l}\text { (Costa et } \\
\text { al., 2016) }\end{array}$ & $\begin{array}{l}\text { (Costa et } \\
\text { al., 2016) }\end{array}$ & $\begin{array}{l}\text { (Costa et al., } \\
\text { 2016; Costa et } \\
\text { al., 2021b) }\end{array}$ & \\
\hline Antibacteriana (Moduladora) & $\begin{array}{l}\text { (Costa et al., } \\
\text { 2016) }\end{array}$ & $\begin{array}{l}\text { (Costa et al., } \\
\text { 2016) }\end{array}$ & $\begin{array}{l}\text { (Costa et } \\
\text { al., 2016) }\end{array}$ & $\begin{array}{l}\text { (Costa et } \\
\text { al., 2016) }\end{array}$ & $\begin{array}{l}\text { (Costa et al., } \\
2016)\end{array}$ & \\
\hline Antifúngica & & & & & $\begin{array}{l}\text { (Costa et al., } \\
\text { 2021b) }\end{array}$ & \\
\hline $\begin{array}{l}\text { Anti- } \\
\text { inflamatória/Antinociceptiva }\end{array}$ & $\begin{array}{l}\text { (Silva et al., } \\
\text { 2015) }\end{array}$ & $\begin{array}{l}\text { (Santiago et al., } \\
\text { 2015) }\end{array}$ & & & & \\
\hline Ansiolítica & $\begin{array}{l}\text { (Araújo et al., } \\
\text { 2018) }\end{array}$ & & & & & \\
\hline Antioxidante & $\begin{array}{l}\text { (Araújo et al., } \\
\text { 2016; Nunes et } \\
\text { al., 2014, 2015) }\end{array}$ & $\begin{array}{l}\text { (Nunes et al., } \\
\text { 2014; Santiago et } \\
\text { al., 2015) }\end{array}$ & $\begin{array}{l}\text { (Nunes et } \\
\text { al., 2014) }\end{array}$ & $\begin{array}{l}\text { (Nunes et } \\
\text { al., 2014) }\end{array}$ & $\begin{array}{l}\text { (Nunes et al., } \\
\text { 2014) }\end{array}$ & $\begin{array}{l}\text { (Nunes et } \\
\text { al., 2014) }\end{array}$ \\
\hline Antiparasitária & & $\begin{array}{l}\text { (Mafud et al., } \\
\text { 2018) }\end{array}$ & & & $\begin{array}{l}\text { (Mafud et al., } \\
\text { 2018) }\end{array}$ & $\begin{array}{l}\text { (Mafud et } \\
\text { al., 2018) }\end{array}$ \\
\hline Antiprotozoário & $\begin{array}{l}\text { (Araújo et al., } \\
\text { 2016) }\end{array}$ & & & & $\begin{array}{l}\text { (Costa et al., } \\
\text { 2021a) }\end{array}$ & \\
\hline Antitumoral & $\begin{array}{l}\text { (Araújo et al., } \\
\text { 2016) }\end{array}$ & & $\begin{array}{l}\text { (Nunes et } \\
\text { al., 2014) }\end{array}$ & $\begin{array}{l}\text { (Nunes et } \\
\text { al., 2014) }\end{array}$ & $\begin{array}{l}\text { (Nunes et al., } \\
2014 \text { ) }\end{array}$ & $\begin{array}{l}\text { (Nunes et } \\
\text { al., 2014) }\end{array}$ \\
\hline Imunomoduladora & & & & & $\begin{array}{l}\text { (Costa et al., } \\
\text { 2021a) }\end{array}$ & \\
\hline
\end{tabular}

Fonte: Elaborado pelos autores baseada nos artigos citados.

\section{Discussão}

Os estudos referentes às riparinas A-F, estão relacionados principalmente a pesquisa dos efeitos bioativos. Uma das primeiras pesquisas publicadas que avaliou uma possível atividade farmacológica dos análogos sintéticos da A. riparia foi o estudo de Nunes e seus colaboradores (2014). A maioria dos estudos que avaliaram estes efeitos foram por meio de experimentos in vitro, desenvolvidos por pesquisadores da Universidade Federal do Piauí em parceira com algumas outras instituições de ensino, representando assim um total de $70 \%$ de todas as publicações.

Nunes e colaboradores (2014), buscaram avaliar o efeito antioxidante das riparinas, através do teor TBARS, níveis de radicais hidroxilas e ensaios de formação de óxido nítrico, das Rips A-F, em experimentos in vitro. Na quantificação do nível de TBARS mostrou que as Rips A-F exercem uma ação antioxidante significativa contra radicais peroxila em todas as concentrações testadas, protegendo os lipídios da oxidação, também sequestraram óxido nítrico (NO). Uma vez que houve uma diminuição significativa na produção desse composto. Tais resultados confirmam o potencial antioxidante in vitro dessas substâncias (Nunes et al., 2014).

Em outro estudo conduzido em 2015, avaliou-se o estresse oxidativo em mitocôndrias isoladas de cérebro e fígado de camundongos tratados com Rip-A, bem como os efeitos sobre a atividade locomotora e a capacidade miorrelaxante. In vitro a substância foi capaz de reduzir significativamente os níveis de radicais livres mesmo em baixas doses. Nos testes in vivo realizados, campo aberto e rota-rod, a Rip-A foi capaz de atravessar a barreira hematoencefálica, entretanto, não causou efeito sedativos e nem perda da coordenação motora ou relaxamento muscular, desta forma desprovida de efeitos colaterais no sistema nervoso central. O estresse oxidativo está diretamente relacionado a doenças crônicas, por exemplo, as cardiovasculares e neurológicas (Nunes et al., 2015).

O efeito antioxidante foi demonstrado utilizando outras metodologias, como analisado pela medição de glutationa (GSH) e malondialdeído (MDA), o GSH é um antioxidante endógeno enzimático e o MDA é gerado por processos lipoperoxidativos, os resultados demonstram que a Rip-B, é capazes de aumentar os níveis de GSH e diminuir a concentração de MDA (Santiago et al., 2015). 
Nos experimentos conduzidos por Araújo e seus colaboradores (2016), a Rip-A mostrou limitada capacidade antioxidante. A oxidação acontece em reações bioquímicas naturais nas células para a produção de energia, na sinalização intercelular e fagocitose. Contudo, o excesso de oxidação pode causar danos às células gerando agravamento de doenças (Appolinário et al., 2011). Têm-se interesse em produtos farmacológicos que promovam proteção contra distúrbios celulares causados pelo estresse oxidativo, uma vez que, a produção excessiva de radicais livres pode oxidar e danificar lipídeos celulares, proteínas e DNA, inibindo a sua função normal e conduzindo a várias doenças (Valko et al., 2007).

Em estudos in vivo, as Rip-A e Rip-B mostraram propriedades anti-inflamatórias e antinociceptiva, a inflamação está ligada a efeitos danosos vasculares e celulares, encontrados em um ambiente de intenso estresse oxidativo. Os modelos experimentais com roedores indicaram que as substâncias inibiram o dano vascular, a produção de citocinas pró-inflamatórias (Fator de Necrose Tumoral alfa - TNF- $\alpha$ e Interleucina 1 beta - IL-1 $\beta$ ) nos locais afetados, modulando o processo de recrutamento de leucócitos e adesão de células endoteliais (Santiago et al., 2015; Silva et al., 2015).

A resposta inflamatória pode ser amplificada pela produção intensa de espécies reativas de oxigênio (ROS), levando a uma redução dos compostos antioxidantes. Nesse contexto, as riparinas possivelmente atuariam reduzindo o estresse oxidativo por meio do aumento dos níveis de GSH. Outra possibilidade é que esse aumento seja secundário a uma diminuição na produção de radicais livres (níveis de TBARS). Além disso, um ponto interessante observado nos experimentos foi a redução induzida do edema de pata, que pode estar associado à regulação negativa da degranulação dos mastócitos pela estabilização de sua membrana (Araújo et al., 2018; Santiago et al., 2015).

Nunes e colaboradores (2014) avaliaram a potencial tumorigênico das Rips A-F, onde citotoxicidade foi avaliada utilizando três linhagens de células tumorais HL-60 (leucêmica), HEP-2 (laríngea) e NCIH-292 (pulmonar), na qual as riparinas C, D, E e F apresentaram ação tumorigênica, com valores de IC $_{50}$ (metade da Concentração Inibitória Máxima) variando de 1,9 a $11,4 \mu \mathrm{g} / \mathrm{mL}$, especialmente em células leucêmicas. Também foi testado o potencial citotóxico das Rips A-F por meio da liberação de LDH, este é um parâmetro utilizado para avaliar o dano celular e tecidual. As Rips apresentaram taxa de inibição de crescimento celular superior a 70\%, sugerindo que essas moléculas possuem provável propriedade antitumoral.

Em outra pesquisa utilizando outras linhagens de células cancerosas, foi avaliado o efeito antitumoral da Rip-A em três linhagens de células neoplásicas de cólon, ovário e glioblastoma. A Rip-A mostrou moderada atividade antiproliferativa com IC50 21,7 $\mu \mathrm{g} / \mathrm{mL}$, com capacidade de inibição de crescimento superior a 90\% em células de adenocarcinoma do cólon (HT-29), esta atividade citotóxica das riparinas parece associar-se com os substituintes de seus anéis aromáticos, e a presença da hidroxila e ausência de grupo metoxi eleva este efeito nas Rips C, D e E (Araújo et al., 2016).

Neste mesmo estudo, a Rip-A também demonstrou atividade leishmanicida moderada sobre as formas promastigostas de Leishmania amazonenses, com valores de $\mathrm{IC}_{50}$ inferiores ao apresentado pelo medicamento padrão antimônio $\mathrm{N}$-metilglucamina para L. amazonesis. N-metil-glucamina pode causar diversos efeitos adversos, além de ser inadequada para o tratamento de mulheres grávidas e pacientes com doenças pulmonares, tuberculose, malária, doenças cardíacas, doenças renais, doenças hepáticas e doença de Chagas, exigindo rigorosa e avaliação e monitoramento constantes no uso clínico. Associado a efeitos colaterais proeminentes, drogas antimoniais também são capazes de induzir resistência parasitária (Araújo et al., 2016).

Outros achados semelhantes aos encontrados por Araújo e colaborados (2014), foram os de Costa et al., (2021a) que testou a atividade antileishmania contra duas formas evolutivas de L. amazonesis e imunomoduladora da Rip-E. A Rip-E causou efeito inibitório contra as formas promastigotas inferior as amastigotas (internalizadas), mostrando que além da atividade direta nas células leishmania, a molécula desempenhou um papel de ativação de macrófagos (imunomoduladora), por meio do aumento fagocitose e maior atividade lisossomal. Avaliou-se a citotoxicidade da Rip-E contra células hospedeiras de mamíferos, com intuito de verificar a seletividade da ação citotóxica. Os resultados mostraram uma maior seletividade para células de Leishmania 
do que para macrófagos de mamíferos, esse achado é importante, pois sugere menos toxicidade no tratamento da leishmaniose (Costa et al., 2021a).

A atividade antiparasitária das Rips A-F foi testada contra vermes de Schistosoma mansoni em experimentos onde avaliou-se a manutenção do ciclo de vida dele, aptidão reprodutiva, produção de ovos e atividade antiesquistossômica. As riparinas $\mathrm{B}, \mathrm{E}$ e F apresentaram atividade contra esquistossomas adultos, todavia as Rips A, C e D foram consideradas inativas. Os parasitas mostraram extensa destruição da superfície externa tegumentar com o aparecimento de inchaço, descamação e erosão de superfície, somados a estes resultados as análises em in sílico das Rips A-F revelaram um perfil promissor para uso por via oral. Demonstrando serem alvos importantes para pesquisa e desenvolvimento de novos fármacos anti-helmíntico (Mafud et al., 2018).

A atividade antimicrobiana in vitro foi testada para as riparinas A, B, C, D e E em cepas de Staphylococcus aureus que superexpressam a bomba de efluxo NorA, apresentando fenótipo de resistência as fluorquinolonas. Nos testes de microdiluição, as riparinas A e B não mostraram atividade antibacteriana intrínseca, entretanto, com o aumento da lipofilicidade houve o aumento da atividade para a Rip-D (Concentração inibitória mínima (MIC) $256 \mu \mathrm{g} / \mathrm{mL} ; \log$ P 2,95), rip-C (MIC $102 \mu \mathrm{g} / \mathrm{mL}$; Log P 3,22) e Rip-E (MIC $16 \mu \mathrm{g} / \mathrm{mL}$; Log P 3,57). Desta forma, a Rip-E pode ser agente promissor anti-estafilococos (Costa et al., 2016).

Todas as riparinas analisadas apresentaram atividade moduladora, ou seja, aumentaram o efeito antimicrobiano das fluorquinolonas (norfloxacina e ciprofloxacino) contra cepas que superexpressam a NorA. Destacando-se a Rip-B que mostrou atividade moduladora para brometo de etídio (substrato conhecido de NorA). Sendo assim, a Rip-B pode ser combinada com fluoroquinolonas, a fim de devolver a essas substâncias a capacidade de tratar bactérias que se tornaram resistentes a elas pela superexpressão da bomba de efluxo NorA. Tornando-se uma estratégia inovadora para o tratamento de infecções bacterianas com esse fenótipo de resistência (Costa et al., 2016).

No estudo in silico, por meio de docagem molecular entre Rip-B e a bomba de efluxo NorA (ligante-receptor), os resultados mostraram que a orientação da Rip-B no local de ligação no receptor estende-se para a porção hidrofóbica, o mesmo local de ligação de outros inibidores NorA (Costa et al., 2016).

Corroborando com esses achados, em outro estudo realizado com as Rip-A e E, analisou-se as MIC e a concentração mínima microbicida (CMM) contra cepas de bactérias gram-positivas (Staphylococcus aureus, S. epidermidis) e gram-negativas (Escherichia coli; Salmonella Typhimurium e Pseudomonas aeruginosa) e fungos (Candida albicans e C. tropicalis). Nesta pesquisa, a Rip-A foi inativa contra todas as cepas de bactérias e fungos testadas, com valores de MIC superior a $1.024 \mu \mathrm{g} / \mathrm{mL}$. A Rip-E mostrou atividade antibacteriana contra cepas de gram-positivas e gram-negativas, com exceção das cepas de Pseudomonas aeruginosa (MIC $1.024 \mu \mathrm{g} / \mathrm{mL}$ ). Além disso, demonstrou atividade antifúngica contra cepas fúngicas analisadas (Costa et al., 2021b).

Um agente antimicrobiano é denominado microbicida se a MMC não for maior que quatro vezes a MIC, a proporção de MMC/MIC encontrada para Rip-E contra cepas sensíveis variou de 3, 2 a 1, demonstrando que a molécula pode ser considerada bactericida e fungicida. O possível mecanismo microbicida foi analisado através de marcação com 7-AAD (7Aminoactinomycin D), relacionado ao dano da membrana plasmática o que pode levar a morte celular. Os valores de MIC de Rip-E necessários para danificar a membrana plasmática bacteriana foi maior nas cepas gram-negativas ( $256 \mu \mathrm{g} / \mathrm{mL})$ do que nas cepas gram-positivas $(32 \mu \mathrm{g} / \mathrm{mL}$ ) (Costa et al., 2021b).

O potencial ansiolítico da Rip-A foi demonstrado utilizando os testes de labirinto em cruz elevado, caixa clara-escura, enterramento em mármore e a segurança toxicológica analisada por meio de teste pré-clínicos de toxidade aguda oral. A análise de docagem molecular revelou que a Rip-A interage com o receptor GABA principalmente nas subunidades $\alpha-2$ e $\beta-1$ e com forte interação com o resíduo de aspartato na posição 68 da subunidade $\beta-1$ (Araújo et al., 2018). 
As pesquisas mencionadas trouxeram como resultados os potenciais farmacológicos dos análogos sintéticos das riparinas obtidas da A. riparia. Atividades antioxidantes (Araújo et al., 2016; Nunes et al., 2014, 2015; Santiago et al., 2015), antitumoral (Araújo et al., 2016; Nunes et al., 2014), leishmanicida (Araújo et al., 2016; Costa et al., 2021a), antibacteriana e moduladora (Costa et al., 2016; Costa et al., 2021b), antifúngica (Costa et al., 2021b), anti-inflamatória e antinociceptiva (Santiago et al., 2015; Silva et al., 2015), esquistossomicida (Mafud et al., 2018), imunomoduladora (Costa et al., 2021b) e ansiolítica (Araújo et al., 2018), foram descritas nos estudos.

Através dessa síntese de dados, aos quais estão disponíveis na literatura, foi possível atribuir propriedades promissoras à bioprospecção de produtos com a utilização das Rips A-F. Pois apresentaram potencial farmacológico para o tratamento de diversas afecções patológicas, ensejando a necessidade de continuidade desses estudos, ao qual são de interesse da sociedade pelo seu impacto social no possível aumento do arsenal terapêutico dos tratamentos de agravos à saúde, para os quais as riparinas já demonstraram atividade em prospecções iniciais de seus efeitos bioativos.

A disponibilidade de novas moléculas com potencial farmacológico é um grande desafio, especialmente na conversão de produtos bioativos em medicamentos. Ao usar produtos naturais com estas propriedades como ponto de partida para o desenvolvimento de novos fármacos, a indústria farmacêutica se depara, em geral, com características não promissoras para a produção de medicamentos, como a baixa concentração das substâncias selecionadas nas fontes naturais, ou solubilidade limitada em meio aquoso, o que por vezes inviabiliza o uso dessas fontes originais (Balunas \& Kinghorn, 2005; Costa, 2009).

Neste contexto, destaca-se as modificações de moléculas, derivadas de plantas medicinais, para a obtenção de novas estruturas com atividade farmacológica e menos efeitos adversos que são o foco de várias pesquisas que pretendem viabilizar a sua aplicação terapêutica. A síntese destas substâncias e de derivados planejados, permite frequentemente que se estabeleça o farmacóforo e que se module o perfil biológico, representando uma excelente oportunidade para a atuação de químicos orgânicos sintéticos e químicos medicinais (Costa, 2009; Carvalho et al., 2013).

Ficou evidenciado que os derivados sintéticos da A. riparia, possuem grande capacidade de tornam-se o protótipo de desenvolvimento de diversos novos medicamentos, com as mais diversas propriedades terapêuticas.

\section{Conclusão}

Os derivados sintéticos, as Rips A-F, mostraram possuir diversas possíveis atividades farmacológicas importantes com potencial para futuras abordagens terapêuticas. Desta forma, são promissoras candidatas para o desenvolvimento de pesquisas voltadas para descobertas de novos fármacos. Entretanto, é nítido a reduzida exploração dessas substâncias pela comunidade científica, isso sugere que é necessário um incremento científico e tecnológico, como também um aprofundamento das pesquisas in vitro, ex vivo, in vivo e clínicas com o intuito embasar a construção literatura cientifica necessária para o desenvolvimento de um futuro medicamento baseados nas riparinas.

\section{Referências}

Appolinário, P. P., Derogis, P. B. M. C., Yamaguti, T. H., \& Miyamoto, S. (2011). Metabolismo, oxidação e implicações biológicas do ácido docosahexaenoico em doenças neurodegenerativas. Química Nova, 34(8), 1409-1416. https://doi.org/10.1590/S0100-40422011000800021

Araújo, É. J. F. de, Lima, L. K. F., Silva, O. A., Júnior, L. M. R., Gutierrez, S. J. C., Carvalho, F. A. de A., Lima, F. das C. A., Pessoa, C., Freitas, R. M. de, \& Ferreira, P. M. P. (2016). In vitro antioxidant, antitumor and leishmanicidal activity of riparin A, an analog of the Amazon alkamides from Aniba riparia (Lauraceae). Acta Amazonica, 46(3), 309-314. https://doi.org/10.1590/1809-4392201505436

Araújo, É. J. F. de, Rezende-Júnior, L. M., Lima, L. K. F., Silva-Júnior, M. P. da, Silva, O. A., Sousa Neto, B. P. de, Almeida, A. A. C. de, Gutierrez, S. J. C., Tomé, A. da R., Lopes, L. da S., Ferreira, P. M. P., \& Lima, F. das C. A. (2018). Pathophysiological investigations, anxiolytic effects and interaction of a semisynthetic riparin with benzodiazepine receptors. Biomedicine \& Pharmacotherapy = Biomedecine \& Pharmacotherapie, 103, 973-981. https://doi.org/10.1016/j.biopha.2018.04.130

Atanasov, A. G., Zotchev, S. B., Dirsch, V. M., \& Supuran, C. T. (2021). Natural products in drug discovery: advances and opportunities. Nature Reviews Drug Discovery, 20(3), 200-216. https://doi.org/10.1038/s41573-020-00114-Z 
Balunas, M. J., \& Kinghorn, A. D. (2005). Drug discovery from medicinal plants. Life Sciences, 78(5), 431-441. https://doi.org/10.1016/j.lfs.2005.09.012 Barbosa Filho, J. M., Silva, E. C., \& Bhattacharyya, J. (1990). Synthesis of Several New Phenylethylamides of Substituted Benzoic Acids. Química Nova, 13(4), $332-334$

Carvalho, A. M. R., Rocha, N. F. M., Vasconcelos, L. F., Rios, E. R. V., Dias, M. L., Silva, M. I. G., de França Fonteles, M. M., Filho, J. M. B., Gutierrez, S. J. C., \& de Sousa, F. C. F. (2013). Evaluation of the anti-inflammatory activity of riparin II (O-methil-N-2-hidroxi-benzoyl tyramine) in animal models. ChemicoBiological Interactions, 205(3), 165-172. https://doi.org/10.1016/j.cbi.2013.07.007

Castelo-Branco, U. V., Castelo-Branco, U. J. V., Thomas, G., Araújo, C. C. de, \& Barbosa-Filho, J. M. (2000). Preliminary Pharmacological Studies on three Benzoyl Amides, constituents of Aniba riparia (Nees) Mez (Lauraceae). Acta Farm. Bonaerense, 19(3), $197-202$.

Catão, R. M. R., Barbosa Filho, J. M., Gutierrez, S. J. C., Lima, E. de O., Pereira, M. do S. V., Arruda, T. A., \& Antunes, R. M. P. (2005). Avaliação da atividade antimicrobiana de riparinas sobre cepas de Staphylococcus aureus e Escherichia coli multirresistentes. Rev. Bras. Anal. Clin, $247-249$.

Costa, L. M., Alves, M. M. de M., Brito, L. M., Abi-Chacra, E. de A., Barbosa-Filho, J. M., Gutierrez, S. J. C., Barreto, H. M., \& Carvalho, F. A. de A. (2021a). In vitro antileishmanial and immunomodulatory activities of the synthetic analogue riparin E. Chemico-Biological Interactions, 336, 109389. https://doi.org/10.1016/j.cbi.2021.109389

Costa, L. M., Macedo, E. V, Oliveira, F. A. A., Ferreira, J. H. L., Gutierrez, S. J. C., Peláez, W. J., Lima, F. C. A., de Siqueira Júnior, J. P., Coutinho, H. D. M., Kaatz, G. W., de Freitas, R. M., \& Barreto, H. M. (2016). Inhibition of the NorA efflux pump of Staphylococcus aureus by synthetic riparins. Journal of Applied Microbiology, 121(5), 1312-1322. https://doi.org/10.1111/jam.13258

Costa, L. M., Sousa, J. N., Braz, D. C., Ferreira, J. H. L., Nogueira, C. E. S., Barbosa-Filho, J. M., Lima-Neto, J. S. S., Gutierrez, S. J. C., Abi-chacra, É. A., \& Barreto, H. M. (2021b). Mechanism of the lethal effect of Riparin E against bacterial and yeast strains. Microbial Pathogenesis, 157, 104968. https://doi.org/10.1016/j.micpath.2021.104968

Costa, P. R. R. (2009). Natural products as starting point for the discovery of new bioactive compounds: Drug candidates with antiophidic, anticancer and antiparasitic properties. Revista Virtual de Química, 1(1), 58-66. https://doi.org/10.5935/1984-6835.20090008

Gutierrez, S. J. C. (2006). Síntese do bowdenol um dihidrobenzofuranoide isolado de Bowdichia virgilioides e preparação de derivados da riparina isolada de Aniba riparia com potencial atividade biológica. Tese de doutorado. Universidade Federal da Paraiba, João Pessoa, PB, Brasil. http://www.dominiopublico.gov.br/pesquisa/DetalheObraForm.do?select_action=\&co_obra=149843

Gutierrez, S. J. C., Claudino, F. de S., Da Silva, B. A., Câmara, C. A., de Almeida, R. N., de Souza, M. de F. V., Da Silva, M. S., Da-Cunha, E. V. L., \& BarbosaFilho, J. M. (2005). Nb-benzoyltryptamine derivatives with relaxant activity in guinea-pig ileum. Il Farmaco, 60(6-7), 475-477. https://doi.org/10.1016/j.farmac.2005.04.001

Hofer, O., Greger, H., Robien, W., \& Werner, A. (1986). 13C NMR and 1H lanthanide induced shifts of naturally occurring alkamides with cyclic amide moieties -amides from falcata. Tetrahedron, 42(10), 2707-2716. https://doi.org/10.1016/S0040-4020(01)90557-5

Mafud, A. C., Silva, M. P. N., Nunes, G. B. L., de Oliveira, M. A. R., Batista, L. F., Rubio, T. I., Mengarda, A. C., Lago, E. M., Xavier, R. P., Gutierrez, S. J. C., Pinto, P. L. S., da Silva Filho, A. A., Mascarenhas, Y. P., \& de Moraes, J. (2018). Antiparasitic, structural, pharmacokinetic, and toxicological properties of riparin derivatives. Toxicology in Vitro: An International Journal Published in Association with BIBRA, 50, 1-10. https://doi.org/10.1016/j.tiv.2018.02.012

Marques, C. A. (2001). Importância econômica da família lauraceae Lindl. FLORAM, 8(núnico), $195-206$.

Nascimento, O., Espírito-Santo, R., Opretzka, L., Barbosa-Filho, J., Gutierrez, S., Villarreal, C., \& Soares, M. (2016). Pharmacological Properties of Riparin IV in Models of Pain and Inflammation. Molecules, 21(12), 1757. https://doi.org/10.3390/molecules21121757

Newman, D. J., \& Cragg, G. M. (2020). Natural Products as Sources of New Drugs over the Nearly Four Decades from 01/1981 to 09/2019. Journal of Natural Products, 83(3), 770-803. https://doi.org/10.1021/acs.jnatprod.9b01285

Nunes, G. B. L., Costa, L. M., Gutierrez, S. J. C., Satyal, P., \& de Freitas, R. M. (2015). Behavioral tests and oxidative stress evaluation in mitochondria isolated from the brain and liver of mice treated with riparin A. Life Sciences, 121, 57-64. https://doi.org/10.1016/j.1fs.2014.11.018

Nunes, G. B. L., Policarpo, P. R., Costa, L. M., da Silva, T. G., Militão, G. C. G., Câmara, C. A., Barbosa Filho, J. M., Gutierrez, S. J. C., Islam, M. T., \& de Freitas, R. M. (2014). In vitro antioxidant and cytotoxic activity of some synthetic riparin-derived compounds. Molecules (Basel, Switzerland), 19(4), 45954607. https://doi.org/10.3390/molecules19044595

Santiago, R. F., de Brito, T. V., Dias, J. M., Dias, G. J., da Cruz, J. S., Batista, J. A., Silva, R. O., Souza, M. H. L. P., de Albuquerque Ribeiro, R., Gutierrez, S. J. C., Freitas, R. M., Medeiros, J.-V. R., \& dos Reis Barbosa, A. L. (2015). Riparin B, a Synthetic Compound Analogue of Riparin, Inhibits the Systemic Inflammatory Response and Oxidative Stress in Mice. Inflammation, 38(6), 2203-2215. https://doi.org/10.1007/s10753-015-0203-4

Silva, R. O., Damasceno, S. R. B., Silva, I. S., Silva, V. G., Brito, C. F. C., Teixeira, A. É. A., Nunes, G. B. L., Camara, C. A., Filho, J. M. B., Gutierrez, S. J. C., Ribeiro, R. A., Souza, M. H. L. P., Barbosa, A. L. R., Freitas, R. M., \& Medeiros, J. V. R. (2015). Riparin A, a compound from Aniba riparia, attenuate the inflammatory response by modulation of neutrophil migration. Chemico-Biological Interactions, 229, 55-63. https://doi.org/10.1016/j.cbi.2015.01.029

Torres, J. M., \& Chavez, A. G. (2001). Secamidas en plantas: distribuición e importancia. Avance Perspective, 20, $377-387$.

Valko, M., Leibfritz, D., Moncol, J., Cronin, M. T. D., Mazur, M., \& Telser, J. (2007). Free radicals and antioxidants in normal physiological functions and human disease. The International Journal of Biochemistry \& Cell Biology, 39(1), 44-84. https://doi.org/10.1016/j.biocel.2006.07.001 